\title{
Effect of a Five-week Scapular Correction Exercise in Patients with Chronic Mechanical Neck Pain
}

\author{
Kang-Seong Lee \\ Department of Biomedical Engineering Welfare Technology, Hanseo University, Seosan, Korea
}

Purpose: The purpose of this study was to compare the change in pain, Neck Disability Index score, and the craniovertebral angle by performing scapular correction exercise or general neck exercise for five weeks in participants with mechanical neck pain.

Methods: A total of 31 participants were randomly assigned between the scapular correction exercise and the general neck exercise groups, and all participants performed intervention for 40 minutes each, three times a week for five weeks The effects were evaluated by measuring the Visual Analog Scale score, the Neck Disability Index score, and the craniovertebral angle, before and after the intervention. Independent t-tests were used to compare differences between two groups, and to compare differences between pre- and post-intervention, paired t-tests were used.

Results: As measured before and after the intervention, the scapular correction exercise group showed significant improvement in all variables $(p<0.05)$, while the general neck exercise group improved only in the neck disability index score. The differences between the two groups revealed further improvement in the scapular correction exercise group compared to the general neck exercise group $(\mathrm{p}<0.05)$.

Conclusion: We found that five weeks of the scapular correction exercise to modify the position and movements of the scapula is clinically an important treatment tool for recovery from chronic mechanical neck pain symptoms and restoration of proper neck function.

Keywords: Scapular correction exercise, Neck pain, Craniovertebral angle

\section{INTRODUCTION}

The definition of mechanical neck pain has not been clearly defined, 1 although it often refers to the pain from the nuchal line to the thoracic vertebrae ${ }^{1}$ and the shoulders. It occurs predominantly as a result of repeated neck movements or consistent neck postures, which results in functional limitations in everyday life. Workers and patients with mechanical pain had misaligned cervical spine and thoracic vertebrae, neck pain and decreased range of motion (ROM) in the joints, and local pain in the muscles surrounding the scapulae. These symptoms reduce the quality of life. ${ }^{2}$

Changes in the cervical spine alignment, such as the forward head position, affect the movement or the position of the scapulae and thoracic vertebrae. ${ }^{3}$ For people who frequently work on computers, it has been reported that the head shifts forward and the po-

Received Apr 3, 2020 Revised Apr 9, 2020

Accepted Apr 14, 2020

Corresponding author Kang-Seong Lee

E-mail cpo114@hanseo.ac.kr sition of the scapulae becomes protracted and rotates downward., 4 Inadequate alignment of the cervical spine and scapulae leads to imbalances in the axioscapular muscles and changes in the lengthtension relationship. This can concentrate the mechanical stress on the cervical spine, which causes stiffness and pain around the neck. ${ }^{6,7}$ Therefore, existing studies on mechanical neck pain have investigated the altered musculoskeletal system between the thoracic vertebrae and scapulae, in addition to the changes in the cervical spine.

Scapular orientation is known to orient the scapulae according to the suitable location of the glenoid fossa. ${ }^{8}$ Scapular alignment generally makes the vertebral border become parallel to the spine, $7 \mathrm{~cm}$ away from the centerline of the spine, and is located between thoracic spine 2 (T2) to T7 or T9. It is rotated 30 degrees to 45 degrees inward on the frontal plane and 10 degrees to 20 degrees tilted for-

Copylight $(02020$ The Korean Society of Physical Therapy

This is an Open Access article distribute under the terms of the Creative Commons Attribution Non-commercial License (https:// creativecommons.org/license/by-nc/4.o.) which permits unrestricted non-commercial use, distribution, and reproduction in any medium, provided the original work is properly cited. 
ward. ${ }^{9-11}$ In previous studies, position correction exercises for the placement of the scapulae were primarily used to solve shoulder problems. ${ }^{12}$ In a recent study, the passive correction of scapular positioning decreased neck pain and improved neck rotation in patients with neck pain. ${ }^{13}$ Scapular postural correction strategy applied to participants with work-related neck pain demonstrated alterations in trapezius activity levels. ${ }^{13}$ These findings suggest that modification of scapular alignment can reduce mechanical stress in the cervical region. Therefore, optimal scapular alignment was considered as an important goal of rehabilitation programs in patients with neck pain.

There have been a few studies regarding the effect of scapular correction exercise for patients with mechanical neck pain as a clinically important protocol. ${ }^{13,14}$ However, there is a lack of evidence to support the importance of this protocol, and most studies have particularly demonstrated the immediate effects of the exercise in patients with neck pain. Therefore, it is necessary to determine the effectiveness of long-term scapular correction exercise for mechanical neck pain. The purpose of this study is to evaluate the change in pain, Neck Disability Index (NDI) score, and the craniovertebral angle by performing the scapular correction exercise or general neck exercise for five weeks in participants with mechanical neck pain, and to compare the differences in the two exercise groups.

\section{METHODS}

\section{Subjects}

In a preliminary study, power analysis was performed using the results of the craniovertebral angle from four participants to calculate the sample size of the participants. As a result of setting the significance level at 0.05, power level at 0.80, and effect size at 0.95, the calculations were induced as a total from 30 participants, with 15 in each group (G-power software 3.1.2, Franz Faul, University of Kiel, Kiel, Germany). A total of 34 participants with mechanical neck pain were recruited and randomly divided into two groups of 17. One group performed a scapular correction exercise (SCE) while the other performed a general neck exercise (GNE). One participant from the SCE group and two participants from the GNE group were eliminated, which reduced the number of participants in the study group to 31 . The inclusion criteria were as follows: 1) age between 18 and 60 years, 2) complaints of pain for at least a 3-month duration, 3) a $\geq 20$ pain rating on a $100-\mathrm{mm}$ visual analog scale (VAS) in the study, 4) a score of at least 10 on the NDI, ${ }^{15}$ 5) pain exacerbated by neck movement or palpation of cervical muscles, and 6) no history of any therapeutic intervention during the past 3 months. $^{16}$

Participants were excluded if they had 1) history of whiplash injury, 2) cervical surgery, 3) previous history of a significant trauma, 4) fracture of the cervical or scapular, 5) neurological impairments, and 6) cervicogenic headache. All participants agreed to join the study and provided informed consent.

\section{Experimental methods}

This study was conducted with a pretest-posttest control group design, and patients with mechanical neck pain were randomly assigned into one of two groups. The main investigator taught the exercise protocols to two physical therapists who had a minimum of five years of experience. In order to minimize the bias between physical therapists, one therapist each was allocated solely to the SCE and GNE groups, respectively. The participants engaged in a total of 15 exercise sessions, three times a week for five weeks.

\section{1) Measurements}

VAS measures the subjective pain level of participants in units of $100 \mathrm{~mm}$, and the reliability of VAS correlated with musculoskeletal pain and disability is known to be excellent $(\mathrm{r}=0.60$ to 0.77$){ }^{17}$

NDI consists of 10 items: pain intensity, personal care, lifting, reading, headache, concentration, work, sleeping, driving, and recreation. Each item consists of six score levels that range from zero to five points. ${ }^{15}$ Higher scores reflect higher disability caused by neck pain, while scores below 15 indicate mild or moderate pain. The intraclass correlation coefficient (ICC) value of test-retest reliability was 0.927 , and concurrent validity with VAS was $r=0.489 .{ }^{15}$

The craniovertebral (CV) angle is suitable to measure the position and the posture of the head.18 A recent study demonstrated reduction in CV angles to produce larger forward neck position. ${ }^{19}$ Furthermore, a smaller CV angle could lead to more severe pain for individuals with neck pain. ${ }^{19} \mathrm{CV}$ angle was measured in this study using Image J v1.46 (National Institutes of Health, Bethesda, MD, USA). Participants had markers to indicate the location of the C7 spinous process, and the side view of their sagittal plane in a comfortable standing position was taken with a digital camera. CV an- 


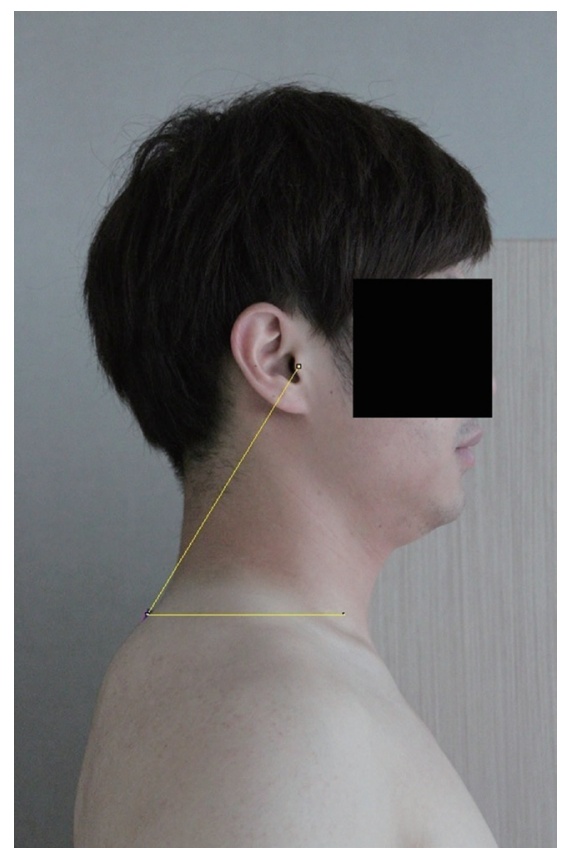

Figure 1. The craniovertebral (CV) angle.

gle refers to the angle created between the horizontal line passing through the spinous process of $\mathrm{C} 7$ and the line passing through the tragus of the ear and the spinous process of $\mathrm{C} 7$ (Figure 1). The interrater and intrarater reliabilities of $\mathrm{CV}$ angle were high $(\mathrm{ICC}=0.88$ to 0.99$).{ }^{20}$

\section{2) Intervention}

All participants partook in one of the two exercises, the SCE or the GNE, for 40 minutes each, three times a week for five weeks. The same five-minute deep cervical flexor training to improve cervical spine stability and the ability to maintain postural function while standing was included in the exercise for both groups. ${ }^{21}$

Participants in the group performing the SCE received assessment of their scapular positions and posture in sitting, lateral, and prone positions. A few paired scapular movements were identified: upward and downward rotation, external and internal rotation, posterior and anterior tilting, elevation and depression, and protraction and retraction. The neutral resting position of the scapula was known as follows: the superior angle (the level of T2 or T3), the scapular spine (T3 or T4), the inferior angle (T7-T9), anterior tilt (1020 degrees), and internal rotation (30 degrees). ${ }^{9-11}$ PT assessed various faulty scapula positions and changes in individuals before exercise. For example, winging of the scapula means excessive down- ward rotation, and an inferior angle protruding means that the anterior tilt is excessive. In addition, it can be seen that the position of the scapula becomes downward rotation, internal rotation, and anterior tilt when soft resistance is applied during a shoulder elevation of 30-40 degrees. ${ }^{11,22}$ The physical therapist (PT) determined the faulty position and movement of the scapula and provided feedback to the participants by hand and speech to guide their positions and to help them learn the movements. The PT stood beside or behind the participants, held the scapular bone with one hand and the upper arm of the same side with the other hand, and corrected the position using passive-active movements. ${ }^{22}$ Furthermore, the physiotherapist used strategies such as palpation, pressure, and resistance in each direction to help participants maintain the correct position. ${ }^{14}$ The entire routine of correcting the position and exercising continued for 35 minutes.

As an example of SCE, the forward tilted scapula was attempted to be corrected into the neutral position through motion feedback via forward and/or backward tilt. The downward-rotated scapula was corrected to the neutral position by using upward rotation. As another example, the protracted and retracted scapular postures were corrected by abducting the arm of the participant to 130 to 140 degrees in the lateral position and setting the scapular upward rotation within the suitable range. Once the participants acquired skills to independently maintain the correct posture, the position and movement of the scapula was modified by teaching them a different posture or raising the arms differently. Many feedbacks were given initially; however, less feedback was provided with better skill acquisition, and the level of difficulty was set accordingly.

GNE comprised passive and active movements of the neck, isometric resistance exercise, and stretching. Passive and active neck movements were performed for 10 minutes in the range of motions that did not incur pain, and isometric resistance exercise was performed for 15 minutes within all possible motion ranges using the treatment band. Stretching was applied to neck muscles, such as the cervical flexors, extensors, and lateral flexors, for 10 minutes. ${ }^{23,24}$

\section{Statistical analysis}

All data were analyzed using SPSS 18.0 (SPSS, Chicago, IL, USA). One-sample Kolmogorov-Smirnov tests were used to confirm the normality of dependent variables. Independent t-tests were used to determine whether there were inter-group differences prior to the 
intervention, and to compare differences between groups. Differences between pre- and post-intervention were determined using paired t-tests. p-values of $<0.05$ indicated statistical significance.

\section{RESULTS}

There were no significant differences in neck pain duration, age, height, and weight between the two groups (Table 1). As a result of pre- and post-test comparison, NDI scores showed significant changes in both groups $(\mathrm{p}<0.001)$. VAS and CV angles were only significantly different in the SCE group $(\mathrm{p}<0.001)$. The GNE group did not show statistically significant differences in VAS $(\mathrm{p}>0.05)$ and $\mathrm{CV}$ angle $(\mathrm{p}>0.05)$. In comparing the differences between the two groups, the SCE group was found to change much more significantly in all measurement variables compared to the GNE group $(\mathrm{p}<0.05)($ Table 2$)$.

\section{DISCUSSION}

This study compared the effectiveness of SCE and GNE in improving pain, functional disability, and head-neck position in people with chronic mechanical neck pain. In particular, it was evident that the SCE performed over five weeks was more effective than the GNE

Table 1. General characteristics of the subjects

\begin{tabular}{lccc}
\hline Variables & SCE group & GNE group & $\mathrm{p}$ \\
\hline Gender (female:male) & $6: 10$ & $5: 10$ & \\
Duration of neck pain (month) & $7.19 \pm 4.61$ & $7.13 \pm 3.48$ & 0.97 \\
Age (yr) & $28.37 \pm 1.78$ & $28.13 \pm 2.33$ & 0.74 \\
Height $(\mathrm{cm})$ & $167.00 \pm 7.94$ & $165.80 \pm 7.90$ & 0.67 \\
Weight $(\mathrm{kg})$ & $60.75 \pm 10.43$ & $60.40 \pm 14.46$ & 0.93 \\
\hline
\end{tabular}

Mean \pm SD

SCE: scapular correction exercise, GNE: general neck exercise. for neck pain, functional recovery and head-to-neck alignment.

Participants with neck pain identified changes in the muscle activity near the scapula and neck, ${ }^{5}$ denoting that changes in muscle activity of scapulothoracic regions such as the trapezius muscles and levator scapulae were correlated to neck and shoulder pain and incorrect posture. ${ }^{4,25}$ In particular, the group that participated in the SCE showed greater improvement than the group that performed the GNE. SCE may have corrected the misaligned scapula and resolved muscle tone imbalance caused by the varying lengths of muscles around the scapulothoracic region.

Pain experienced on the neck and areas surrounding the neck is one of the factors that increase stress and decrease function and quality of life for workers and patients. ${ }^{26}$ An increase in the NDI score, which is a self-reported measurement tool, signifies an improvement in the level of disability from chronic neck pain and the functional levels to accomplish daily living activities. As a result of a systematic review to evaluate the effects of exercise on individuals with chronic neck pain, strength training, stretching, and shoulderthoracic stability exercises demonstrated improvement in function through an increase in NDI scores. ${ }^{27}$ According to a recent study that compared the effects of neck exercises to the effects of neck exercises performed combined with scapulae stabilization exercises, both groups improved in neck pain and NDI scores; however, there was no difference between the groups. ${ }^{24}$ This study showed significant improvement in NDI scores in both SCE and GNE groups, and the former particularly had greater improvements than the latter. Therefore, it was found that the group that performed the neck exercise with the SCE had improved levels of disability and function; thus, the neck exercise with the SCE served as an effective protocol for neck pain improvement compared to the neck exercises alone.

The position of the head for individuals with neck pain is corre-

Table 2. Comparison of NDI, VAS, CV angle between and within two groups

\begin{tabular}{|c|c|c|c|c|c|c|c|}
\hline \multirow{2}{*}{ Variables } & \multicolumn{3}{|c|}{ SCE group } & \multicolumn{3}{|c|}{ GNE group } & \multirow{2}{*}{$p$} \\
\hline & Pre-test & Post-test & $p$ & Pre-test & Post-test & $\mathrm{p}$ & \\
\hline NDI (score) & $16.50 \pm 3.20$ & $12.31 \pm 2.87$ & $<0.001^{*}$ & $16.00 \pm 2.20$ & $14.40 \pm 2.50$ & $<0.001^{*}$ & $<0.001^{*}$ \\
\hline VAS & $66.56 \pm 9.78$ & $41.86 \pm 11.67$ & $<0.001^{*}$ & $61.33 \pm 8.55$ & $56.33 \pm 6.94$ & 0.051 & $<0.001^{*}$ \\
\hline CV angle $\left({ }^{\circ}\right)$ & $46.74 \pm 4.16$ & $50.29 \pm 3.69$ & $<0.001^{*}$ & $46.86 \pm 4.42$ & $48.23 \pm 4.86$ & 0.051 & $0.014^{+}$ \\
\hline
\end{tabular}

Mean \pm SD.

SCE: scapular correction exercise, GNE: general neck exercise, NDI: neck disability index, VAS: visual analog scale. CV: craniovertebral.

${ }^{*} p<0.05$ : $p$ value is comparison between pre- and post-intervention using paired t-test, ${ }^{+} p<0.05$ : $p$ value is comparison between SCE and GNE group using independent t-test. 
lated with the degree of forward inclination. CV angle was smaller than that of healthy adults, and there was a moderately negative correlation with neck disability. ${ }^{19}$ Deep cervical flexion training showed a strong level of evidence for improvement in neuromuscular control. ${ }^{28}$ Additionally, muscle strengthening and stretching of the neck and shoulder area, which was performed to improve the posture of participants with misaligned head position, was known to be effective in posture correction through increased CV angle. ${ }^{29}$ Similar to the results of previous studies, ${ }^{27,28}$ the deep cervical flexion training, applied to the SCE and GNE groups as a baseline, improved the function of the head and neck posture by increasing the CV angle. In particular, the SCE, which modified the scapular movement and posture, strengthened the weakened stability muscles and affected the recovery of shortened muscles, and this is assumed to have increased the CV angle in comparison to the GNE, which only focused on the neck.

This study demonstrated that five weeks of SCE performed on participants with mechanical neck pain was effective in reducing pain, functional impairment, and correcting the head-neck position. Moreover, the SCE group had greater pain reduction, recovery of function, and improved head-neck position compared to the GNE group. Therefore, exercises that modify the position and movement of the scapula have been suggested to be effective in improving mechanical neck pain.

The limitation of this study was the lack of follow-up assessment for long-term effect observations. Furthermore, if variables such as kinematic data of the cervical spine, muscle activity, and quality of life were added, it could have been used as a strong basis to explain the findings. Therefore, further studies will be required to establish the rationale for the clinical effectiveness of SCE.

\section{REFERENCES}

1. Fernandez de Las Penas C, Simons D, Cuadrado ML et al. The role of myofascial trigger points in musculoskeletal pain syndromes of the head and neck. Curr Pain Headache Rep. 2007;11(5):365-72.

2. Jun $D$. A longitudinal investigation of the moderating effect of social support on job strain developing non-specific neck pain in office workers. J Kor Phys Ther. 2019;31(4):254-9.

3. Finley MA, Lee RY. Effect of sitting posture on 3-dimensional scapular kinematics measured by skin-mounted electromagnetic tracking sensors. Arch Phys Med Rehabil. 2003;84(4):563-8.

4. Szeto GP, Straker L, Raine S. A field comparison of neck and shoulder postures in symptomatic and asymptomatic office workers. Appl Ergon. 2002;33(1):75-84.

5. Falla D, Farina D. Muscle fiber conduction velocity of the upper trapezius muscle during dynamic contraction of the upper limb in patients with chronic neck pain. Pain. 2005;116(1-2):138-45.

6. Lee H, Song J. Functioning characteristics of patients with neck pain: ICF concept based. J Kor Phys Ther. 2019;31(4):242-7.

7. Falla D, Jull G, Russell T et al. Effect of neck exercise on sitting posture in patients with chronic neck pain. Phys Ther. 2007;87(4):408-17.

8. Mottram SL. Dynamic stability of the scapula. Man Ther. 1997;2(3):12331.

9. Kim EK, Lee DK. Effect of scapular brace on the pulmonary function and foot pressure of elderly women with forward head posture. J Kor Phys Ther. 2018;30(4):141-5.

10. Kendall FP, McCreary EK, Provance PG et al. Muscles: Testing and Function, with posture and pain. Baltimore, MD: Lippincott Williams \& Wilkins. 2005:51-85.

11. Jull G, Sterling M, Falla D et al. Whiplash, headache and neck pain: research based directions for the physical therapies. Elsevier. 2008:206-7.

12. Ludewig PM, Cook TM. Alterations in shoulder kinematics and associated muscle activity in people with symptoms of shoulder impingement. Phys Ther. 2000;80(3):276-91.

13. Ha SM, Kwon OY, Yi CH et al. Effects of passive correction of scapular position on pain, proprioception, and range of motion in neck-pain patients with bilateral scapular downward-rotation syndrome. Man Ther. 2011;16(6):585-9.

14. Wegner S, Jull G, O'Leary S et al. The effect of a scapular postural correction strategy on trapezius activity in patients with neck pain. Man Ther. 2010;15(6):562-6.

15. Vernon H, Mior S. The Neck Disability Index: a study of reliability and validity. J Manipulative Physiol Ther. 1991;14(7):409-15.

16. Munoz Munoz S, Munoz Garcia MT, Alburquerque Sendin F et al. Myofascial trigger points, pain, disability, and sleep quality in individuals with mechanical neck pain. J Manipulative Physiol Ther. 2012;35(8): 608-13.

17. Boonstra AM, Schiphorst Preuper HR, Reneman MF et al. Reliability and validity of the visual analogue scale for disability in patients with chronic musculoskeletal pain. Int J Rehabil Res. 2008;31(2):165-9.

18. Hickey ER, Rondeau MJ, Corrente JR et al. Reliability of the cervical range of motion device and plumb line techniques in measuring resting head posture. J Man Manip Ther. 2000;8(1):10-7.

19. Yip CH, Chiu TT, Poon AT. The relationship between head posture and severity and disability of patients with neck pain. Man Ther. 2008;13(2): 148-54.

20. Kang D, Oh T. Comparison of the muscle activity in the normal and forward head postures based on the pressure level during cranio-cervical flexion exercises. J Kor Phys Ther. 2019;31(1):1-6.

21. Falla D, O'Leary S, Fagan A et al. Recruitment of the deep cervical flexor muscles during a postural-correction exercise performed in sitting. Man Ther. 2007;12(2):139-43.

22. Mottram SL, Woledge RC, Morrissey D. Motion analysis study of a scapular orientation exercise and subjects' ability to learn the exercise. Man Ther. 2009;14(1):13-8.

23. Phadke A, Bedekar N, Shyam A et al. Effect of muscle energy technique 
and static stretching on pain and functional disability in patients with mechanical neck pain: A randomized controlled trial. Hong Kong Physiother J. 2016;35:5-11.

24. Yildiz TI, Turgut E, Duzgun I. Neck and scapula-focused exercise training on patients with nonspecific neck pain: A randomized controlled trial. J Sport Rehabil. 2018;27(5):403-12.

25. Hanvold TN, Waersted M, Mengshoel AM et al. The effect of work-related sustained trapezius muscle activity on the development of neck and shoulder pain among young adults. Scand J Work Environ Health. 2013;39(4):390-400.

26. Rezai M, Côté P, Cassidy JD et al. The association between prevalent neck pain and health-related quality of life: a cross-sectional analysis. Eur Spine J. 2009;18(3):371.

27. Gross A, Kay TM, Paquin JP et al. Exercises for mechanical neck disorders. Cochrane Database Syst Rev. 2015;1:CD004250.

28. Blomgren J, Strandell E, Jull G et al. Effects of deep cervical flexor training on impaired physiological functions associated with chronic neck pain: a systematic review. BMC Musculoskelet Disord. 2018;19(1):415.

29. Xu L, Hwang B, Kim T. The Effect of postural correction and visual feedback on muscle activity and head position change during overhead arm lift test in subjects with forward head posture. J Kor Phys Ther. 2019; 31(3):151-6. 